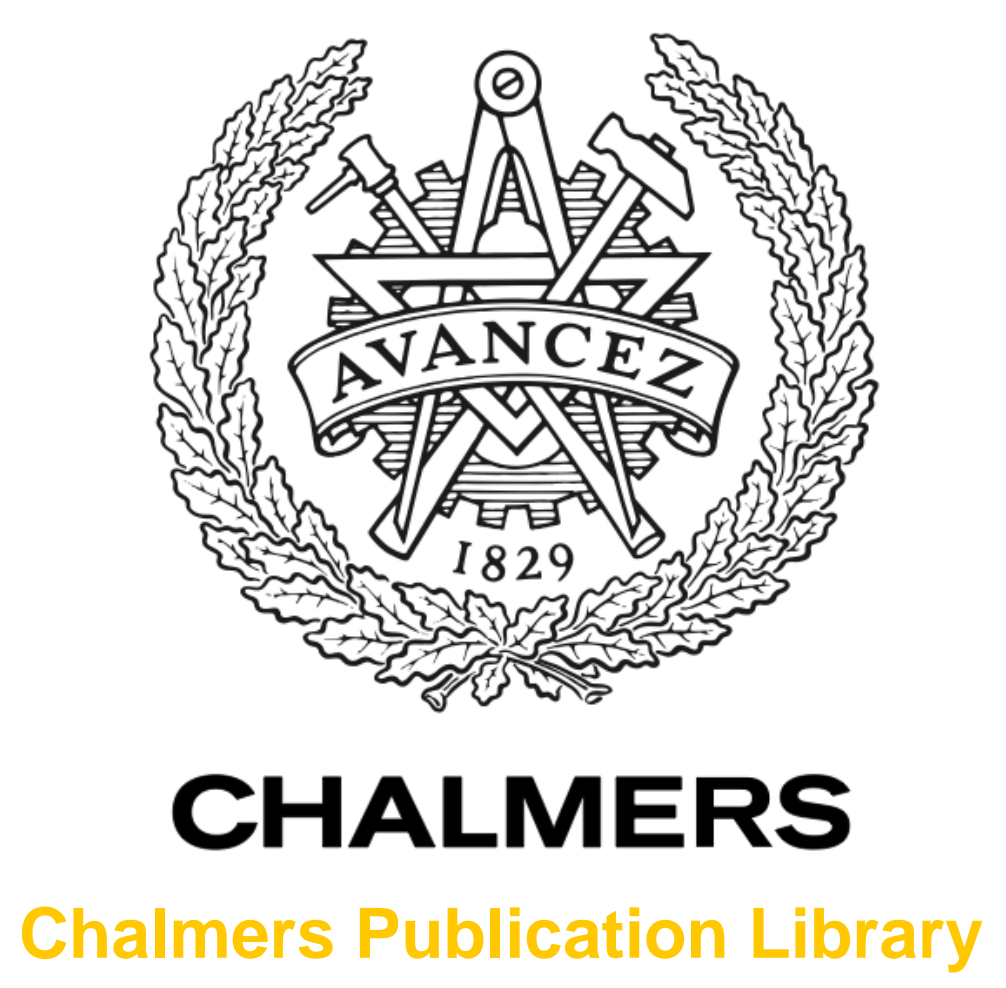

\title{
On Medium Access and Physical Layer Standards for Cooperative Intelligent Transport Systems in Europe
}

This document has been downloaded from Chalmers Publication Library $(\mathrm{CPL})$. It is the author's version of a work that was accepted for publication in:

Proceedings of the IEEE (ISSN: 0018-9219)

Citation for the published paper:

Ström, E. (2011) "On Medium Access and Physical Layer Standards for Cooperative Intelligent Transport Systems in Europe". Proceedings of the IEEE, vol. 99(7), pp. 11831188 .

http://dx.doi.org/10.1109/jproc.2011.2136310

Downloaded from: http://publications.lib.chalmers.se/publication/143365

Notice: Changes introduced as a result of publishing processes such as copy-editing and formatting may not be reflected in this document. For a definitive version of this work, please refer to the published source. Please note that access to the published version might require a subscription. 


\title{
On Medium Access and Physical Layer Standards for Cooperative Intelligent Transport Systems in Europe
}

\author{
Erik G. Ström, Senior Member, IEEE
}

\begin{abstract}
In this paper, we will outline the current European development of wireless communications to support cooperative Intelligent Transport Systems (ITS). The focus will be on spectrum allocation and the physical and medium access control layers of the ITS-G5 access technology, which is under specification by the European Telecommunications Standards Institute (ETSI). The European ITS architecture and European standardization will also be briefly discussed.
\end{abstract}

\section{INTRODUCTION}

Cooperative intelligent transport systems have been subject to numerous research projects in Europe as well as globally (see [1] for an extensive list of projects and organizations). With cooperative systems, we mean systems in which vehicles and infrastructure cooperates to solve safety, efficiency, or other tasks. Cooperation is only possible if the involved entities can communicate, and wireless communications is, of course, required when vehicles are involved. Although infrared communications is also considered for ITS systems, we will here only discuss radio systems.

In this paper, we will focus on the latest developments in Medium Access Control (MAC) and Physical (PHY) layer research and standardization for the European market. This includes a brief discussion of important European standardization bodies, e.g., European Committee for Standardization (CEN), European Committee for Electrotechnical Standardization (CENELEC), and European Telecommunications Standards Institute (ETSI).

An extensive overview of the current efforts in Europe can be found in 198-page document [2]. The document is a deliverable from the coordinate support action COMeSafety, http://www.comesafety.org, whose goal is to "support realization and possible deployment of cooperative, communicationbased active safety systems." The European ITS architecture, described in detail in [2] and summarized by Kosch, et al., in [3], is similar to the traditional OSI layered model for communications with the application layer at the top and the link and physical layers at the bottom, see Fig. 1.

The bottom layer in the European ITS architecture, namely "Access Technologies," has the purpose to interface with the different communication technologies that are available in an ITS station. An access technology should contain at least the link and physical layers, but could also be a global navigation satellite system such as Galileo or GPS or a complete

E. G. Ström is with the Chalmers University of Technology, Gothenburg, Sweden.

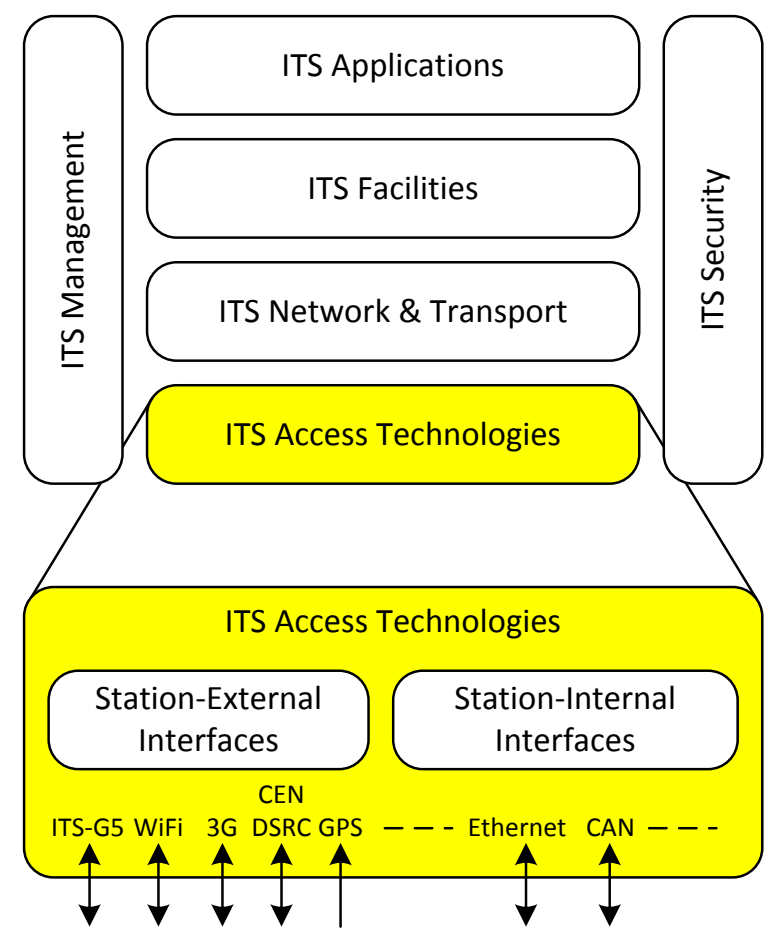

Fig. 1. European ITS Architecture [2]

communication system (with a full protocol stack), such as UMTS, WiMAX, or WiFi. An access technology could also be for station-internal communication, e.g., Ethernet or Controller Area Network (CAN), a very common wired bus in vehicles.

The access technology that is most relevant for this Special Issue is ETSI ITS-G5, which is a European profile of IEEE $802.11 \mathrm{p}$. It is the only radio technology that allows for direct vehicle-to-vehicle and vehicle-to-roadside communications (i.e., without usage of a central controller or access point) in a peer-to-peer configuration. As such, it has the potential to support very low latency communications and is therefore a crucial enabling technology for cooperative ITS applications with strict delay requirements.

In Europe, the term Dedicated Short-Range Communication (DSRC), refers to a short-range communication technology standardized by CEN. CEN-DSRC allows for direct vehicleroadside communications in a master-slave configuration and is used mainly for electronic toll collection applications. In the US and Australia, DSRC refers to systems based on IEEE 802.11p, and in Japan, DSRC refers to a system that 
originally targeted similar applications as CEN-DSRC and is quite different from $802.11 \mathrm{p}$. The fact that DSRC can refer to three different systems has caused much confusion during the years.

The remainder of this paper is organized as follows. In the next section, we will list abbreviations for quick reference. Important organizations for European standardization are highlighted in Sec. III. The European ITS spectrum allocation is described in Sec. IV, and ETSI ITS-G5 is the topic of Sec. V. Finally, the paper is concluded in Sec. VI.

\section{ABbreviations And TERMinology}

\begin{tabular}{|c|c|}
\hline 3GPP & The 3rd Generation Partnership Project \\
\hline BRAN & Broadband Radio Access Networks \\
\hline BSS & Basic Service Set \\
\hline CAN & Controller area network \\
\hline CEN & European Committee for Standardization \\
\hline CENELEC & $\begin{array}{l}\text { European Committee for Electrotechnical } \\
\text { Standardization }\end{array}$ \\
\hline CEPT & $\begin{array}{l}\text { European Conference of Postal and Telecom- } \\
\text { munications Administrations }\end{array}$ \\
\hline DFS & Dynamic Frequency Selection \\
\hline DSRC & Dedicated Short-Range Communication \\
\hline ECC & $\begin{array}{l}\text { Electronic Communications Committee, a } \\
\text { business committee of CEPT }\end{array}$ \\
\hline EIRP & Equivalent Isotropic Radiated Power \\
\hline $\mathrm{EN}$ & European Norm (European Standard) \\
\hline ES & ETSI Standard \\
\hline ESO & European Standardization Organization \\
\hline ETSI & $\begin{array}{l}\text { European Telecommunications Standards In- } \\
\text { stitute }\end{array}$ \\
\hline $\mathrm{FCC}$ & Federal Communications Commission \\
\hline GPS & Global Positioning System \\
\hline ITS & Intelligent Transport System \\
\hline ITS-G5 & $\begin{array}{l}\text { set of protocols and parameters specified in } \\
\text { the ESTI Standard ES } 202663 \text { "Intelligent } \\
\text { Transport Systems (ITS); European profile } \\
\text { standard for the physical and medium access } \\
\text { control layer of Intelligent Transport Systems } \\
\text { operating in the } 5 \mathrm{GHz} \text { frequency band." }\end{array}$ \\
\hline ITS-GA & Frequency band from 5.875 to $5.905 \mathrm{GHz}$ \\
\hline ITS-GB & Frequency band from 5.855 to $5.875 \mathrm{GHz}$ \\
\hline ITS-GC & Frequency band from 5.470 to $5.725 \mathrm{GHz}$ \\
\hline MAC & Medium Access Control \\
\hline NSO & National Standards Organization \\
\hline OFDM & Orthogonal Frequency-Division Multiplexing \\
\hline PHY & Physical \\
\hline RLAN & Radio Local Area Network \\
\hline STA & Station \\
\hline STDMA & $\begin{array}{l}\text { Self-Organizing Time-Division Multiple Ac- } \\
\text { cess }\end{array}$ \\
\hline STF & Specialist Task Force \\
\hline TC & Technical Committee \\
\hline UMTS & $\begin{array}{l}\text { Universal Mobile Telecommunications Sys- } \\
\text { tem (an umbrella term for the third generation } \\
\text { radio technologies developed within 3GPP) }\end{array}$ \\
\hline $\mathrm{E}$ & Wireless Access in Vehicular Environments \\
\hline
\end{tabular}

Wi-Fi Brandname of the Wi-Fi Alliance, normally used interchangeably with IEEE 802.11

WiMAX Worldwide Interoperability for Microwave Access, often used interchangeably with IEEE 802.16

WG Work Group

WLAN Wireless Local Area Network

\section{StANDARDIZATION FOR THE EUROPEAN MARKET}

The three main European Standardization Organizations (ESOs), European Committee for Standardization (CEN), European Committee for Electrotechnical Standardization (CENELEC), and European Telecommunications Standards Institute (ETSI), are, in principle, responsible for different subject areas. However, in many fields, such as ITS, collaboration is needed. The ESOs have different types of deliverables, ranging from technical reports to European standards. A European standard (also known as a European Norm [EN]) automatically becomes a national standard in the $27 \mathrm{EU}$ member states, Croatia, and the European Free Trade Association countries (Iceland, Norway, and Switzerland). These countries are bound to remove any conflicting national standards to make room for an EN.

The ITS area is mostly covered by ETSI, but to a lesser degree also by CEN. CENELEC is not really involved in ITS standardization.

The most relevant CEN technical committees are CEN/TC 278 Road Transport and Telematics, which is responsible for, among other things, CEN-DSRC, and CEN/TC 226 Road Equipment, which is in charge of road infrastructure such as traffic lights and variable message signs.

ETSI is made up of more than 700 member organizations from some 60 countries worldwide. The work is mainly carried out in technical committees (TCs), ETSI projects, or ETSI partnership projects. An ETSI Standard (ES) is subject to approval of all ETSI members (also non-European members). However, approval of ENs is restricted to the National Standards Organizations (NSOs) of the 27 European Union countries, Croatia, Iceland, Norway, and Switzerland. There is one NSO per country.

The most ITS-relevant ETSI technical body is, quite naturally, TC ITS. TC ITS is concerned with all types of communications in vehicles, between vehicles, and between vehicles and roadside. Moreover, TC ITS also include the use of information and communication technologies for rail, water and air transport, including navigation systems.

TC ITS consists of five working groups (WGs)

- WG1 User and application requirements

- WG2 Architecture, cross-layer, web services

- WG3 Transport and network

- WG4 Media and medium related issues

- WG5 Security

It is WG4 that is responsible for the development of ITS- 


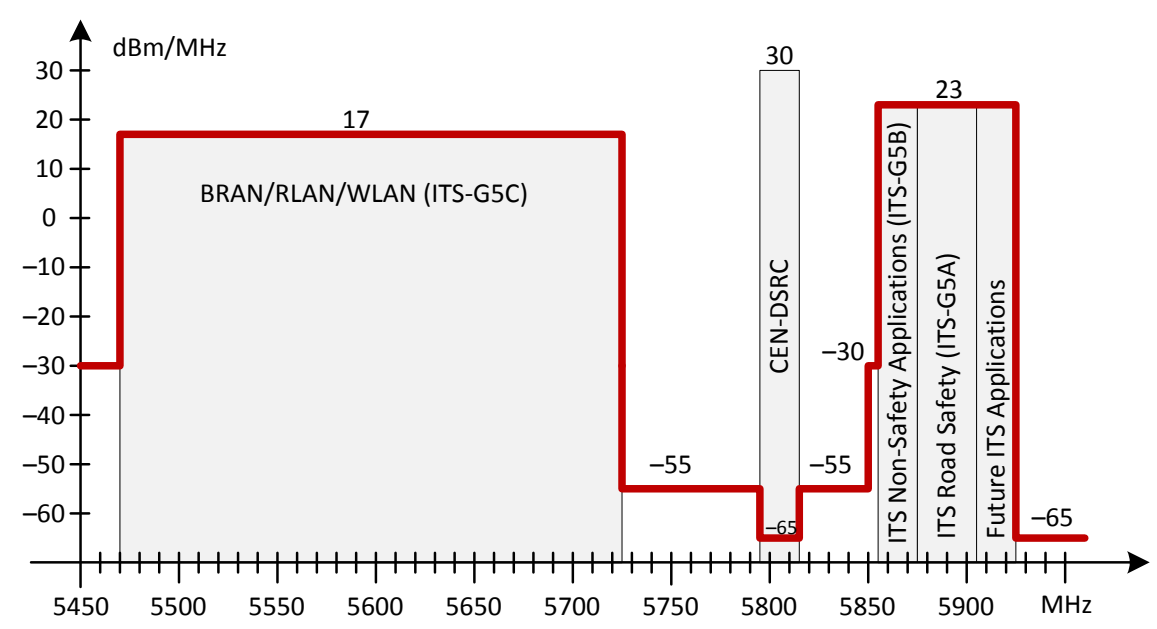

Fig. 2. Maximum limit of mean power spectral density (EIRP) [4].

TABLE I

FREQUENCY ALLOCATION IN THE EUROPEAN UNION [4].

\begin{tabular}{|c|c|c|c|}
\hline Frequency Range [MHz] & Usage & Regulation & Harmonized Standard \\
\hline $5905-5925$ & Future ITS application & ECC Decision [6] & EN 302571 [7] \\
\hline 5 & & ECC Dec & [7] \\
\hline-5875 & ITS non-safety applications & ECC Recommendation [9] & EN 302571 [7] \\
\hline $5470-5725$ & RLAN (BRAN, WLAN) & ERC Decision [10] Commission Decisions [11] and [12] & EN 301893 [13] \\
\hline
\end{tabular}

G5 [4] and the Specialist Task Force ${ }^{1}$ (STF) 395, which are discussed in more detail below.

Input to the standardization in ESOs is provided by EU projects, industry consortia, and other global and regional standardization bodies. Among the most important recent EU projects, we find COMeSafety, Coopers, CVIS, and SAFESPOT [2]. In Europe, the most important industry consortium is the Car-2-Car Communication Consortium [5].

\section{European ITS Spectrum Allocation}

The ITS spectrum allocation in Europe is described in the ETSI standard ES 202633 [4], and summarized in Table I. Similar spectrum bands have been allocated in the US, $75 \mathrm{MHz}$ in the range $5.850-5.925 \mathrm{GHz}$ and in Japan, $80 \mathrm{MHz}$ in the range $5.770-5.850 \mathrm{GHz}$ [2].

Three specific bands are defined in [4]: ITS-G5A, from 5.875 to $5.905 \mathrm{GHz}$, dedicated to ITS for safety related applications; ITS-G5B, from 5.855 to $5.875 \mathrm{GHz}$, dedicated to ITS non-safety applications; and ITS-G5C, from 5.470 to $5.725 \mathrm{GHz}$, which is a Radio Local Area Network (RLAN) band that can be used also for ITS applications. The spectrum mask is found in Fig. 2. It should be noted that the spectrum mask is further constrained, as described below and in [4].

In Europe, CEN-DSRC operates in the band from 5.795$5.805 \mathrm{GHz}$ and in some European countries also in the 5.805$5.815 \mathrm{GHz}$ band, see Fig. 2. The vicinity of this band to the ITS-G5B band has raised concerns about interference between CEN-DSRC and ITS-G5, and co-existence studies

\footnotetext{
${ }^{1}$ ETSI describes an STF as "An STF is a team of highly-skilled experts working together over a pre-defined period to draft an ETSI standard under the technical guidance of an ETSI Technical Body and with the support of the ETSI Secretariat."
}

are therefore on-going in ETSI's in STF 395, which belongs to TC-ITS WG4. The interference concerns are also manifested in the ITS-G5 spectrum mask, see Fig. 2.

The channel spacing in ITS-G5A and ITS-G5B is set to $10 \mathrm{MHz}$, which is the same spacing as in WAVE [14]. Hence, there is room for five channels in ITS-G5A and ITS-G5B: one control channel, G5CC, and four service channels, G5SC1G5SC4 [4]. The control channel and service channels are defined in Table II and depicted in Fig. 3.

As seen from in Fig. 3, the spectrum mask (thick solid line) is not used to its maximum emission limit, presumably to limit interference into the important control channel and to adhere to the strict out-of-band emission limits into the CEN-DSRC band.

The service channel G5SC5 is defined to be in the $255 \mathrm{MHz}$ wide ITS-G5C band, and the channel spacing can be either 10 or $20 \mathrm{MHz}$. The ITS-G5C band is shared with RLAN systems and an ITS station must therefore follow the requirements in [11]-[13]. In particular, a method for Dynamic Frequency Selection (DFS) must be used to reduce interference between RLAN devices and radar systems. Due to this requirement, it is expected that a fixed (roadside) ITS station will act as the DFS master device and that vehicular ITS stations are DFS slave devices. The maximum transmission limits are $30 \mathrm{dBm}$ EIRP or $17 \mathrm{dBm} / \mathrm{MHz}$ for a DFS master and $23 \mathrm{dBm}$ EIRP or $10 \mathrm{dBm} / \mathrm{MHz}$ for a DFS slave (the more stringent requirement prevails in case the EIRP and power density limits are different).

\section{ETSI ITS-G5}

The access technology ITS-G5 is a European profile of IEEE 802.11 and include features of IEEE 802.11p. Hence, 
TABLE II

EUROPEAN CHANNEL ALLOCATION [4].

\begin{tabular}{|c|c|c|c|c|c|}
\hline \multirow[t]{2}{*}{ Band } & \multicolumn{2}{|c|}{ Channel } & \multirow{2}{*}{$\begin{array}{c}\text { Center Frequency } \\
{[\mathrm{MHz}]}\end{array}$} & \multirow{2}{*}{$\begin{array}{l}\text { TX Power Limit } \\
\text { [dBm EIRP] }\end{array}$} & \multirow{2}{*}{$\begin{array}{l}\text { TX Power Density Limit } \\
{[\mathrm{dBm} / \mathrm{MHz}]}\end{array}$} \\
\hline & ETSI & IEEE & & & \\
\hline \multirow[t]{3}{*}{ G5A } & G5CC & 180 & 5900 & 33 & 23 \\
\hline & $\mathrm{G} 5 \mathrm{SC} 2$ & 178 & 5890 & 23 & 13 \\
\hline & G5SC1 & 176 & 5880 & 33 & 23 \\
\hline \multirow[t]{2}{*}{ G5B } & G5SC3 & 174 & 5870 & 23 & 13 \\
\hline & G5SC4 & 172 & 5860 & 0 & -10 \\
\hline
\end{tabular}

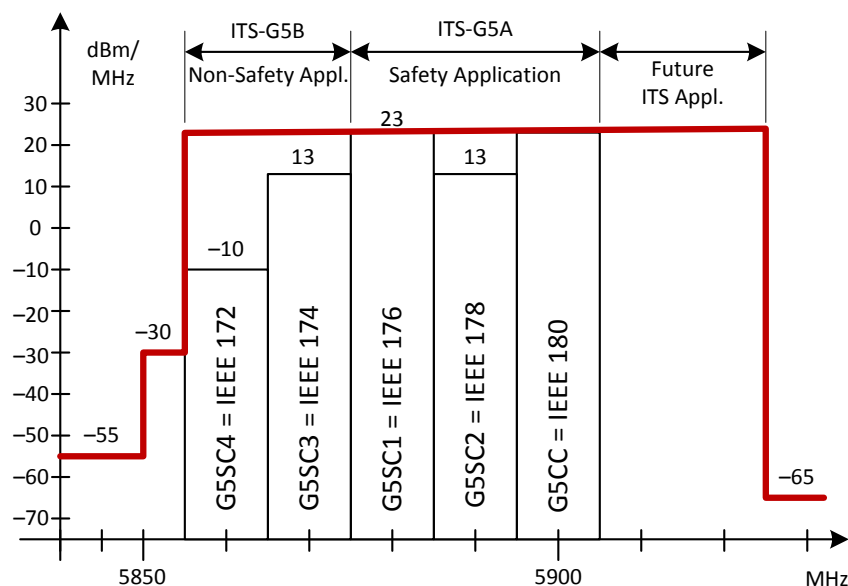

Fig. 3. Channel allocation and spectrum mask for ITS-G5A and ITS-G5B channels.

the ETSI standard document ES 202663 [4] defines the MAC and PHY layers of an ITS-G5 station and relies heavily on 802.11 [15] and the 802.11p amendment [16].

\section{A. MAC layer}

An ITS-G5 station must operate "outside the context of a BSS," [16]. A Basic Service Set (BSS) is essentially a set of 802.11 stations that have recognized each other and established communication ${ }^{2}$. In short, "operating outside the context of a BSS" enables ITS-G5 stations to avoid the authentication and association delays required for establishing a BSS. This will reduce communication latency in networks with rapidly changing topologies, such as vehicular networks. Stations operating on the ITS-G5A and ITS-G5B bands will act as peers, regardless if the stations are fixed or mobile. On the other hand, for stations operating on ITS-G5C must act as either DFS masters or slaves. It is foreseen that fixed ITS stations will be DFS masters and mobile ITS stations will be DFS slaves. This will make it impossible for mobile ITS stations to communicate directly on the ITS-G5C band [4].

An important feature is that all ITS-G5 stations must, when not transmitting, always be able to receive data on the control channel (G5CC). This implies that an ITS station that operates on any of the service channels (the G5SCs), must be able to receive data on both the service and control

\footnotetext{
${ }^{2}$ The definition in [15] is "Basic service set (BSS): A set of stations (STAs) that have successfully synchronized using the JOIN service primitives and one STA that has used the START primitive. Membership in a BSS does not imply that wireless communication with all other members of the BSS is possible."
}

channels simultaneously. Stations that do not support traffic safety applications are, however, exempted from this rule. The dual-channel reception requirement makes power save modes cumbersome, and the 802.11 power management services are therefore not used by ITS-G5 devices. All ITS-G5 stations, regardless if they support traffic safety applications or not, must be able to transmit on the control channel.

The wireless medium is accessed using carrier sensing multiple access with collision avoidance (CSMA/CA), as described in $802.11 \mathrm{p}$ standard [16]. It is well-known that CSMA/CA does not perform well in congested situations. Since there are concerns about congestion on, in particular, the control channel, ETSI's Specialist Task Force 395 are currently studying the applicability of congestion control methods and the more robust medium access scheme Self-Organizing TimeDivision Multiple Access (STDMA) [17].

\section{B. PHY Layer}

The physical layer is 802.11 OFDM [15, Clause 17], i.e., Orthogonal Frequency-Division Multiplexing (OFDM) with 48 data subcarriers, 4 pilot subcarriers, and 12 null subcarriers (64 subcarriers in total). The main timing and frequency parameters are summarized in Table III. For the ITS-G5A and ITS-G5B bands, the channel spacing is $10 \mathrm{MHz}$ and, therefore, the OFDM symbol duration is $T_{\mathrm{OFDM}}=8 \mu \mathrm{s}$, which includes a cyclic prefix (guard interval) of $1.6 \mu \mathrm{s}$. The difference in propagation time of the first and last propagation path (the maximum delay spread) should, ideally, not exceed $1.6 \mu \mathrm{s}$. Expressed as a difference in propagation path length, this corresponds to roughly $(1.6 \mu \mathrm{s}) \cdot\left(3 \times 10^{8} \mathrm{~m} / \mathrm{s}\right) \approx 480 \mathrm{~m}$, which is a reasonable distance compared to the target maximum communications range, which is less than $1 \mathrm{~km}$.

The expected channel time-variations is characterized by the Doppler spread. The maximum Doppler frequency that can affect a direct transmission between two vehicles that are traveling with a relative speed of $v$ is $f_{D}=v f_{c} / c$, where $f_{c}$ is the carrier frequency and $c$ is the electromagnetic propagation speed $^{3}$. A rough estimate of the coherence time, i.e., the time it takes for the channel significantly change (decorrelate) is $1 / f_{D}$. To avoid intercarrier interference in an OFDM system, it is crucial that the OFDM symbol duration is much smaller than the coherence time, i.e., $f_{D} T_{\mathrm{OFDM}} \ll 1$. If we consider a highspeed scenario with relative speed $v=206 \mathrm{~km} / \mathrm{h}=85 \mathrm{~m} / \mathrm{s}$ and assume that $f_{c}=5.9 \mathrm{GHz}$ and $c=3 \times 10^{8} \mathrm{~m} / \mathrm{s}$, then $f_{D} T_{\text {OFDM }} \approx 0.0134$, which indicates that intercarrier

\footnotetext{
${ }^{3}$ If the radio wave interact with other objects on its way from the transmitted to the receiver, the maximum Doppler frequency can actually be larger than $c f_{c} / c$.
} 
TABLE III

802.11 OFDM PARAMETERS FOR 10 AND 20 MHz CHANNEL SPACING [15, ClaUSE 17]

\begin{tabular}{cccc}
\hline Channel Spacing & $\Delta F$ & $20 \mathrm{MHz}$ & $10 \mathrm{MHz}$ \\
Subcarrier Spacing & $\Delta f=\Delta F / 64$ & $312.5 \mathrm{kHz}$ & $156.25 \mathrm{kHz}$ \\
Orthogonality Duration & $T=1 / \Delta f$ & $3.2 \mu \mathrm{s}$ & $6.4 \mu \mathrm{s}$ \\
Cyclic Prefix (Guard Interval) & $T_{\mathrm{GI}}=T / 4$ & $0.8 \mu \mathrm{s}$ & $1.6 \mu \mathrm{s}$ \\
OFDM Symbol Duration & $T_{\mathrm{OFDM}}=T+T_{\mathrm{GI}}$ & $4 \mu \mathrm{s}$ & $8 \mu \mathrm{s}$ \\
\hline
\end{tabular}

interference will not be a serious problem. However, we note that the channel coherence time is roughly $75 T_{\text {OFDM }}$. For the default data rate of $6 \mathrm{Mbits} / \mathrm{s}$, this corresponds to a frame payload of $75 \times 6=450$ bytes (ignoring the preamble overhead), which is comparable to the frame lengths often discussed in the literature (300-800 bytes). Hence, we can expect significant time variations over a frame in high-speed scenarios, and this will pose a serious challenge to the channel estimation algorithms in the receiver.

In the ITS-G5C band, the channel spacing can be either 10 or $20 \mathrm{MHz}$. For the $20 \mathrm{MHz}$ case, all timing parameters are halved compared to the $10 \mathrm{MHz}$ case, see Table III. Hence, if the channel spacing is doubled, the robustness against delay spread is reduced (due to a halved cyclic prefix), while the the robustness against time-varying channel conditions is increased (due to a halved OFDM symbol duration).

\section{SUMMARY AND CONCLUSIONS}

In this paper, we have briefly described the state of European ITS standardization. The European ITS architecture differs from other initiatives, such as WAVE [14], mainly in the higher layers. However, the important access technology ETSI ITSG5 is based on 802.11p, which is also the basis for WAVE.

There are, of course, differences in terminology that could cause confusion. For instance, the WAVE control channel is at channel 178, which is a service channel (G5SC2) in the ETSI terminology, see Fig. 3. Vice versa, the ETSI control channel (G5CC) is a WAVE service channel (channel 180).

Other, perhaps more important, differences follows from the fact that an ITS-G5 station that supports traffic safety applications must listen to the control channel at all times, except when transmitting. Hence, such an ITS-G5 station must be able to decode packets on two separate channels at the same time, which will require two receivers (or a more advanced wide-band receiver) per station. A WAVE station, on the other hand, can use the time-division feature of IEEE 1609.4 to switch back and forth between the WAVE control channel and the WAVE service channels. This would seemingly allow a WAVE station to have only a single receiver. However, channel switching has recently been made optional and it might be that traffic safety messages will be sent without time-division (i.e., at any time) on the service channel 172, and this will require dual receiver stations in some, but not all, application scenarios, see [14] for details.

Future discrepancies, such as special congestion control methods and changes to MAC layer, can result from the ongoing work in ETSI's specialist task force 395.

\section{ACKNOWLEDGEMENTS}

The author would like acknowledge the fruitful discussions with his co-workers Katrin Sjöberg and Elisabeth Uhlemann and the financial support from Chalmers' Area of Advance in Transport.

\section{REFERENCES}

[1] L. Strandén, E. G. Ström, and E. Uhlemann, "Wireless communications vehicle-to-vehicle and vehicle-to-infrastructure," SAFER prestudy no. AD4, Oct. 2008, full text available at https://publications.lib. chalmers.se/cpl/record/index.xsql?pubid=137657.

[2] COMeSafety, "European ITS communication architecture, ver. 3.0," Feb. 2010, http://www.comesafety.org/?id=64.

[3] T. Kosch, I. Kulp, M. Bechler, M. Strassberger, B. Weyl, and R. Lasowski, "Communication architecture for cooperative systems in Europe," IEEE Communications Magazine, vol. 47, no. 5, pp. 116-125, May 2009.

[4] ETSI ES 202663 (V1.1.0): "Intelligent Transport Systems (ITS); European profile standard for the physical and medium access control layer of Intelligent Transport Systems operating in the $5 \mathrm{GHz}$ frequency band".

[5] CAR 2 CAR Communication Consortium, http://www.car-to-car.org/.

[6] ECC/DEC(02)01: "ECC Decision of 15 March 2002 on the frequency bands to be designated for the co-ordinated introduction of Road Transport and Traffic Telematic Systems".

[7] ETSI EN 302571 (V1.1.1): "Intelligent Transport Systems (ITS); Radiocommunications equipment operating in the $5855 \mathrm{MHz}$ to $5925 \mathrm{MHz}$ frequency band; Harmonized EN covering the essential requirements of article 3.2 of the R\&TTE Directive".

[8] Commission Decision 2008/671/EC of 5 August 2008 on the harmonised use of radio spectrum in the $5875-5905 \mathrm{MHz}$ frequency band for safety-related applications of Intelligent Transport Systems (ITS).

[9] ECC/REC/(08)01: "ECC Recommendation (08)01 on the use of the band 5855-5875 MHz for Intelligent Transport Systems (ITS)".

[10] ERC/DEC(99)23: "ERC Decision of 29 November 1999 on the harmonised frequency bands to be designated for the introduction of High Performance Radio Local Area Networks (HIPERLANs)".

[11] Commission Decision 2005/513/EC of 11 July 2005 on the harmonised use of radio spectrum in the $5 \mathrm{GHz}$ frequency band for the implementation of wireless access systems including radio local area networks (WAS/RLANs).

[12] Commission Decision 2007/90/EC of 12 February 2007 amending Decision 2005/513/EC on the harmonised use of radio spectrum in the 5 $\mathrm{GHz}$ frequency band for the implementation of Wireless Access Systems including Radio Local Area Networks (WAS/RLANs).

[13] ETSI EN 301893 (V1.5.1): "Broadband Radio Access Networks (BRAN); $5 \mathrm{GHz}$ high performance RLAN; Harmonized EN covering the essential requirements of article 3.2 of the R\&TTE Directive".

[14] J. B. Kenney, "Dedicated short range communications (DSRC) standards in the United States," Proceedings of the IEEE, 2011, to appear.

[15] IEEE Standard 802.11-2007: Telecommunications and information exchange between systems- Local and metropolitan area networks- Specific requirements-Part 11: Wireless LAN Medium Access Control (MAC) and Physical Layer (PHY) Specifications.

[16] IEEE Standard 802.11p-2010: Telecommunications and information exchange between systems- Local and metropolitan area networksSpecific requirements-Part 11: Wireless LAN Medium Access Control (MAC) and Physical Layer (PHY) Specifications, Amendment 6: Wireless Access in Vehicular Environments.

[17] K. Bilstrup, E. Uhlemann, E. G. Ström, and U. Bilstrup, "On the ability of the 802.11p MAC method and STDMA to support real-time vehicle-to-vehicle communications," EURASIP Journal on Wireless Communications and Networking, 2009, article ID 902414 , doi:10.1155/2009/902414. 